\title{
Hypertrophic obstructive cardiomyopathy: Comparison of outcomes after myectomy or alcohol ablation adjusted by propensity score
}

\author{
Anthony Ralph-Edwards, $\mathrm{MD}^{\mathrm{a}}$ \\ Anna Woo, MD ${ }^{\mathrm{b}}$ \\ Brian W. McCrindle, MD, MPH ${ }^{\mathrm{c}}$ \\ Jonathan L. Shapero, BSc ${ }^{d}$ \\ Leonard Schwartz, MD \\ Harry Rakowski, MD \\ E. Douglas Wigle, $M D^{\mathrm{b}}$ \\ William G. Williams, MD
}

From the Divisions of Cardiovascular Sur-
${\text { gery and Cardiology, }{ }^{\mathrm{a}} \text { Toronto General }}$ Hospital (University Health Network),
Toronto; the Division of Cardiology, The
Hospital for Sick Children, Toronto; and
Queen's University, Kingston, Ontario,
Canada.

Read at the Eighty-fourth Annual Meeting of The American Association for Thoracic Surgery, Toronto, Ontario, Canada, April 25-28, 2004.

Received for publication April 29, 2004; revisions received July 9, 2004; accepted for publication Aug 18, 2004.

Address for reprints: William G. Williams, MD, 555 University Ave, Suite 1525, Toronto, Ontario, Canada M5G1X8 (Email: bill.williams@sickkids.ca).

J Thorac Cardiovasc Surg 2005;129:351-8 $0022-5223 / \$ 30.00$

Copyright (C) 2005 by The American Association for Thoracic Surgery

doi:10.1016/j.jtcvs.2004.08.047
Objective: In November 1998, our center began offering alcohol ablation as an alternative to surgical myectomy for patients with hypertrophic obstructive cardiomyopathy. Patients with concomitant lesions were referred for surgical intervention, and the others were offered either treatment option. We sought to review the early outcomes for both protocols.

Methods: One hundred fifty patients had intervention for hypertrophic obstructive cardiomyopathy to June 30,2003 . Sixty patients elected to have alcohol ablation, and 5 crossed over to surgical intervention. A total of 95 patients had a myectomy. Patients having an isolated myectomy $(\mathrm{n}=48)$ are compared with those who had an ablation. Hospital records were reviewed, and follow-up contact (mean, 2.2 years) with the patient or referring cardiologist and recent echocardiographic reports were obtained. Differences in clinical and hemodynamic outcomes between achieved treatment groups were compared after adjustment for differing baseline patient characteristics, including use of a propensity score, to adjust for the nonrandomization.

Results: The patients undergoing alcohol ablation $(n=60)$ were older (58 vs 48 years) and had fewer associated lesions (1 vs 39 patients), lower pressure gradients (67 vs $73 \mathrm{~mm} \mathrm{Hg}$ ), and similar symptomatic status and degrees of mitral regurgitation compared with those in the myectomy group. Alcohol ablation was abandoned in 6 patients, 5 of whom underwent myectomy. Among the completed alcohol ablations, there were 5 late deaths, and 1 other patient was referred for myectomy. One late death occurred after myectomy. At latest follow-up, 3-year survival is 97\%, and $92 \%$ of the patients are in New York Heart Association class II or I. Adjusted comparisons showed significantly lower postintervention left ventricular outflow gradients at rest in the myectomy group ( $5 \mathrm{vs} 15 \mathrm{~mm} \mathrm{Hg}$ ), with provocation (14 vs $42 \mathrm{~mm} \mathrm{Hg}$ ), mitral systolic anterior motion (67\% vs 29\%), and New York Heart Association class. No significant difference was present in postintervention septal thickness or freedom from postintervention pacing, although in time-related analysis, the 3 -year freedom from pacing is $88 \%$ versus $59 \%(P=.02)$, favoring myectomy.

Conclusion: Either alcohol ablation or myectomy offers substantial clinical improvement for patients with hypertrophic obstructive cardiomyopathy. Hemodynamic resolution of the obstruction and its sequelae is more complete with myectomy. Residual lesions after alcohol ablation might affect longer-term outcomes. 


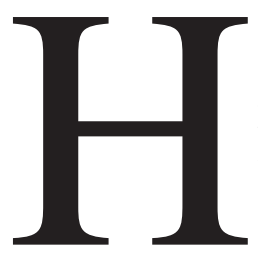

ypertrophic obstructive cardiomyopathy is an inherited disorder with variable expressivity, resulting in asymmetric septal hypertrophy and left ventricular outflow tract obstruction. Symptoms are similar to those of aortic stenosis. Goals of treatment include symptomatic control, resolution of hemodynamic abnormalities and their sequelae, reduction of sudden cardiac death risk, and screening of family members. Left ventricular outflow tract obstruction at rest is a predictor of severe symptoms, heart failure, and death. ${ }^{1}$ The majority of patients are managed medically, predominantly with $\beta$-blockers. Surgical treatment has been limited to patients with refractory symptoms and high resting gradients. Results of surgical intervention are well documented, with $95 \%$ of patients being asymptomatic with accompanying dramatic reduction in outflow tract gradient. ${ }^{2-7}$ Alcohol ablation is a newer percutaneous technique that was first used at our institution in November $1998 .{ }^{8-10}$ It is designed to ablate hypertrophied cardiac septal muscle through localized infarction, but its efficacy compared with that of surgical myectomy is uncertain. ${ }^{11}$

In this communication we compare these 2 treatment modalities using propensity adjustment to compare their effects on symptoms and hemodynamics.

\section{Methods}

At the Toronto General Hospital between November 1998 and December 2003, 150 symptomatic adults with hypertrophic obstructive cardiomyopathy underwent intervention for left ventricular outflow tract obstruction. During this period, 60 patients were referred for alcohol ablation, and 90 patients were referred for surgical myectomy. Operations were performed as described previously. ${ }^{2}$ Alcohol ablation was performed with echocardiographic contrast guidance, as previously described. ${ }^{8-10}$

Myectomy was performed in 90 patients referred directly to surgical intervention and in 5 others after attempted ablation. Among this group of 95 patients, 48 patients had an isolated septal myectomy, and 47 patients had a myectomy and a concomitant procedure. The 54 patients who received alcohol ablation and the 48 surgical patients who had an isolated myectomy were compared with respect to symptom status, echocardiographic parameters of left ventricular outflow tract obstruction and mitral regurgitation, need for additional procedures, and survival.

\section{Data Analysis}

Data are described as frequencies, medians with ranges, and means with SDs. Where there are missing data, the number of nonmissing values is given.

Initial differences in baseline characteristics between achieved treatment groups were sought in bivariable analysis by using $\chi^{2}$ tests, Fisher exact tests, Student $t$ tests, and Kruskal-Wallis analysis of variance. Multiple logistic regression analysis was used to determine independent baseline variables predictive of membership in the ablation group. Multivariable analysis was used after imputation of missing values with the mean value for the com- bined groups to adjust for any differences at baseline between the groups being compared. Multiple logistic regression incorporating all significant baseline variables was used to derive a propensity score or the predicted probability that an individual patient belonged to the ablation group. Comparisons between groups regarding outcome variables was then performed unadjusted, adjusted for significant baseline variables, and adjusted for significant baseline variables and propensity score in multivariable regression analysis. All statistical analyses were performed with SAS statistical software (Version 8; SAS Institute, Inc, Cary, NC).

\section{Results}

\section{Study Subjects}

The study included 155 intended procedures in 150 patients, including 60 intended alcohol ablations and 90 intended surgical myectomies. Of the 60 intended ablations, 54 were completed, including one patient who subsequently went on to have a surgical myectomy because of ongoing symptoms and an important residual gradient. Of the 6 patients who did not have an intended ablation, 5 subsequently had surgical myectomy (however, one patient's successful operation was outside of the study period and was not included), and one patient had no further procedures except pacemaker placement. All 90 intended surgical myectomies were performed. However, added to the surgical group are 4 of the patients who were intended to have ablation but did not, and one patient who had ablation followed by later myectomy, for a total of 95 myectomies. Of the 95 myectomies, 48 were performed as isolated procedures and serve as the group for comparison with the 54 completed ablation procedures.

\section{Comparison of Isolated Surgical Myectomy $(\mathrm{n}=48)$ Versus Completed Alcohol Ablation $(n=54)$}

The preprocedure characteristics of each group are shown in Table 1. In univariate comparisons the patients undergoing alcohol ablation were significantly older, had higher resting systolic blood pressure, were less likely to have angina or palpitations or presyncope-syncope, had better New York Heart Association (NYHA) class, were more likely to have coronary artery disease, and had decreased thickness of the ventricular septum and posterior wall relative to the patients undergoing isolated surgical myectomy. These same factors, except coronary artery disease and septal thickness, independently differentiated the 2 groups in stepwise multiple logistic regression analysis, with a c statistic of 0.908 . These factors, together with missing value flags, were entered in multiple logistic regression analysis to create the propensity score or the probability that a given patient was in the alcohol ablation group.

\section{Duration of Postprocedure Hospitalization}

There were no deaths during the postprocedure hospitalization. Median duration was 8 days (range, 4-37 days) for the alcohol ablation group versus 7 days (range, 3-19 days) for 
TABLE 1. Comparison of baseline characteristics between those undergoing achieved alcohol ablation versus those undergoing surgical myectomy with no concomitant procedures

\begin{tabular}{|c|c|c|c|}
\hline & $\begin{array}{l}\text { Alcohol ablation } \\
\quad(\mathrm{n}=54)\end{array}$ & $\begin{array}{l}\text { Surgical myectomy } \\
\qquad(\mathrm{n}=48)\end{array}$ & $P$ value \\
\hline Male/female sex & 26:28 & 30:18 & .15 \\
\hline Mean age at procedure (y) & $59 \pm 15$ & $46 \pm 17$ & $<.001$ \\
\hline Mean weight at procedure $(\mathrm{kg})$ & $82 \pm 18$ & $85 \pm 18$ & .49 \\
\hline Mean body surface area $\left(\mathrm{m}^{2}\right)$ & $1.90 \pm 0.23$ & $1.98 \pm 0.27$ & .13 \\
\hline Mean resting systolic blood pressure (mm Hg) & $129 \pm 23(52)$ & $118 \pm 16(45)$ & .02 \\
\hline Mean resting diastolic blood pressure $(\mathrm{mm} \mathrm{Hg})$ & $75 \pm 12(52)$ & $72 \pm 11(45)$ & .31 \\
\hline \multicolumn{4}{|l|}{ Symptoms } \\
\hline Dyspnea & $40 / 51(78 \%)$ & $42 / 48(88 \%)$ & .24 \\
\hline Angina & $22 / 51(43 \%)$ & $31 / 48(65 \%)$ & .04 \\
\hline Palpitations & $2 / 51(4 \%)$ & $15 / 48(31 \%)$ & $<.001$ \\
\hline Presyncope-syncope 4/51 (8\%) & $18 / 48(38 \%)$ & $<.001$ & \\
\hline \multicolumn{4}{|l|}{ Medications at procedure } \\
\hline$\beta$-blocker & $35 / 49(71 \%)$ & $30 / 44(68 \%)$ & .74 \\
\hline Disopyramide & $40 / 49(82 \%)$ & $35 / 44(80 \%)$ & .80 \\
\hline Aspirin & $2 / 49(4 \%)$ & $0 / 44(0 \%)$ & .50 \\
\hline Preprocedure coronary artery disease & $9 / 49(18 \%)$ & $0 / 46(0 \%)$ & .003 \\
\hline Muscle bridge of LAD & $2(4 \%)$ & $1(2 \%)$ & 1.00 \\
\hline Preprocedure heart rhythm & $\mathrm{n}=53$ & $\mathrm{n}=45$ & \\
\hline Normal sinus rhythm & $48(91 \%)$ & $42(93 \%)$ & \\
\hline Paced & $5(9 \%)$ & $1(2 \%)$ & \\
\hline Atrial fibrillation & $0(0 \%)$ & $2(5 \%)$ & \\
\hline \multicolumn{4}{|l|}{ Preprocedure echocardiogram } \\
\hline Aortic regurgitation & $\mathrm{n}=53$ & $\mathrm{n}=40$ & \\
\hline None & $29(55 \%)$ & $25(63 \%)$ & \\
\hline Trivial & $16(30 \%)$ & $13(35 \%)$ & \\
\hline Mild & $8(15 \%)$ & $2(5 \%)$ & .22 \\
\hline \multicolumn{4}{|l|}{ Mitral regurgitation } \\
\hline Trivial & $1(2 \%)$ & $4(8 \%)$ & \\
\hline Mild & $14(26 \%)$ & $8(17 \%)$ & \\
\hline Mild to moderate & $5(9 \%)$ & $10(21 \%)$ & \\
\hline Moderate & $18(33 \%)$ & $19(40 \%)$ & \\
\hline Moderate to severe & $13(24 \%)$ & $3(6 \%)$ & \\
\hline Severe & $3(6 \%)$ & $4(8 \%)$ & .34 \\
\hline Systolic anterior motion of MV & $\mathrm{n}=50$ & $\mathrm{n}=47$ & \\
\hline None & $0(0 \%)$ & $1(2 \%)$ & \\
\hline Mild & $11(22 \%)$ & $11(23 \%)$ & \\
\hline Moderate & $15(30 \%)$ & $19(40 \%)$ & \\
\hline Moderate to severe & 0 & $1(2 \%)$ & \\
\hline Severe & $24(48 \%)$ & $15(32 \%)$ & .21 \\
\hline LVOT gradient at rest $(\mathrm{mm} \mathrm{Hg})$ & $74 \pm 36(53)$ & $64 \pm 27(47)$ & .13 \\
\hline Septal thickness (mm) & $22 \pm 5(50)$ & $25 \pm 6(46)$ & .04 \\
\hline LV posterior wall thickness & $11 \pm 3(48)$ & $12 \pm 3(43)$ & $<.05$ \\
\hline LV mass index $\left(\mathrm{g} / \mathrm{m}^{2}\right)$ & $157 \pm 40(45)$ & $165 \pm 52(38)$ & .45 \\
\hline Left atrial size $(\mathrm{mm})$ & $47 \pm 6(48)$ & $47 \pm 8(47)$ & .73 \\
\hline LV end-diastolic dimension (mm) & $43 \pm 5(48)$ & $43 \pm 6(44)$ & .85 \\
\hline LV end-systolic dimension (mm) & $24 \pm 4(48)$ & $23 \pm 7(42)$ & .30 \\
\hline
\end{tabular}

$L A D$, Left anterior descending coronary artery; $M V$, mitral valve; $L V O T$, left ventricular outflow tract; $L V$, left ventricular.

the isolated myectomy group $(P=.46)$. None of the baseline variables were significantly associated with duration of hospitalization. When the groups were compared after pro- pensity adjustment, the alcohol ablation group had significantly longer postprocedure hospitalizations (adjusted estimate, 4.1 days longer; $P=.02$ ). 


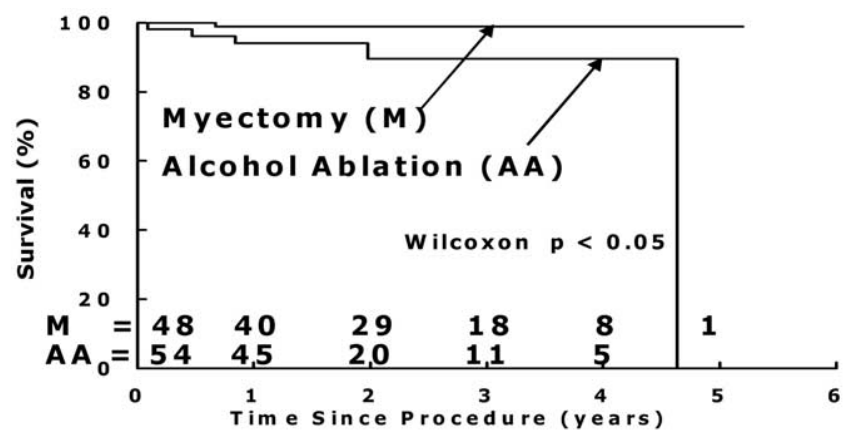

Figure 1. Survival after intervention with alcohol ablation or after isolated myectomy is plotted by using Kaplan-Meier analysis.

\section{Mortality}

During follow-up, there were 5 deaths in the alcohol ablation group ( 2 sudden, 1 congestive heart failure, 1 malignancy, and 1 after liver transplantation). No deaths occurred in the isolated myectomy group. Kaplan-Meier estimates for time-related survival in the alcohol ablation group were $94 \%$ at 1 year and $90 \%$ at 4 years, with one death occurring at 4.6 years after the procedure. This was significantly different from the isolated myectomy group $(P=.02$, Figure 1). Given the small number of events, no baseline variable was significantly associated with time-related survival, and no adjusted analysis was performed.

\section{Pacemaker Placement}

Before the procedure, 6 patients in each group had a permanent pacemaker placement. Excluding these 12 patients, time-related survival free of pacemaker placement was determined for each group (Figure 2) and was significantly greater in the isolated myectomy group in an unadjusted comparison (hazard ratio, 2.65; $P<.05$ ). The only baseline variable significantly associated with an increased risk of time-related pacemaker placement was the presence of coronary artery disease (hazard ratio, 2.98; $P<.05$ ). After adjusting for this variable, the difference in risk of pacemaker placement was no longer statistically significant between the groups (hazard ratio, 2.34; $P=.10$ ), including after additional adjustment with propensity score (hazard ratio, 2.98; $P=.14$ ).

\section{NYHA Class at Latest Follow-up}

NYHA functional class data were available at latest follow-up for 47 patients in the alcohol ablation group and 42 patients in the isolated myectomy group at a mean interval of $2.3 \pm 1.3$ years after the procedure. For the alcohol ablation group, 19 were in class I, 24 were in class II, and 4 were in class III, and for the isolated myectomy group, 34 were in class I, 6 were in class II, and 2 were in class III $(P<.001)$. In ordinal logistic regression the baseline variables significantly associated with worse functional class included older age at the time of the

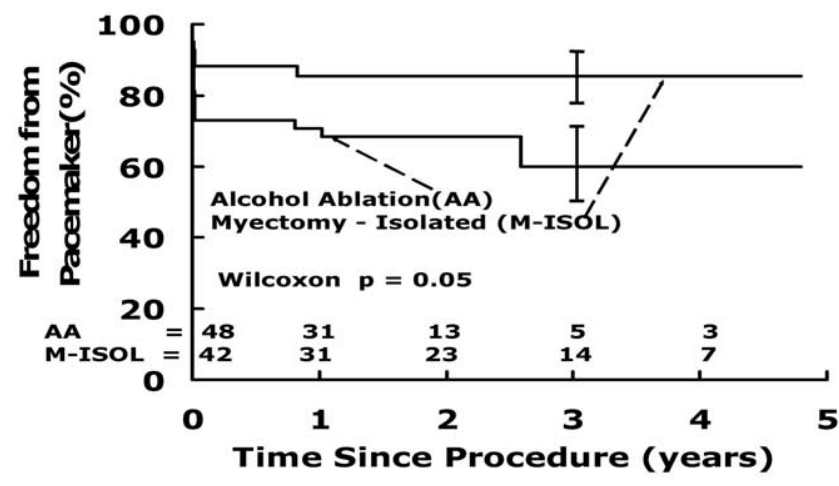

Figure 2. Survival free of a permanent pacemaker implant is shown by using Kaplan-Meier analysis for the ablation and isolated myectomy (M-ISOL) groups. For this analysis, the 6 patients in each group who had a pacemaker before intervention are excluded.

procedure (odds ratio, 1.04; $P=.02$ ), female sex (odds ratio, $3.30 ; P=.02)$, and larger preprocedure size of the left atrium (odds ratio, 1.09; $P=.01$ ). The patients undergoing alcohol ablation had significantly worse functional class after adjustment for these variables (odds ratio, 5.40; $P=.004$ ), and after additional adjustment with propensity score (odds ratio, 5.43; $P=.05)$.

Echocardiographic Assessment at Latest Follow-up Echocardiographic assessments had been performed in all of the patients undergoing alcohol ablation and in 46 of the patients undergoing isolated myectomy at a mean interval of $2.0 \pm 1.3$ years after their procedures (Table 2). Postintervention resting left ventricular outflow gradients were significantly higher in patients who underwent alcohol ablation. In general linear regression modeling, a higher gradient was significantly associated with the baseline variables of older age at procedure and better NYHA functional class. After adjusting for these 2 baseline variables, resting gradient remained significantly higher in the alcohol ablation group (adjusted difference, $14 \mathrm{~mm} \mathrm{Hg} ; P<.001$ ), a difference that persisted after additionally adjusting with propensity score $(P=.002)$. Provoked left ventricular outflow gradients were also significantly higher in patients who underwent alcohol ablation. In general linear regression modeling, a higher provoked gradient was significantly associated with the baseline variables absence of syncope and better NYHA functional class. After adjustment, provoked gradient remained significantly higher in the alcohol ablation group (adjusted difference, $25 \mathrm{~mm} \mathrm{Hg} ; P<.001$ ) and remained so after additional adjustment with propensity score $(P=.004)$.

The presence of systolic anterior motion of the mitral valve (SAM) after intervention was significantly higher in the alcohol ablation group. In multiple logistic regression 
TABLE 2. Comparison of follow-up echocardiographic variables between those undergoing achieved alcohol ablation versus surgical myectomy with no concomitant procedures

\begin{tabular}{|c|c|c|c|c|c|}
\hline & $\begin{array}{l}\text { Alcohol ablation } \\
\quad(n=54)\end{array}$ & $\begin{array}{l}\text { Surgical myectomy } \\
\text { (n = 46) }\end{array}$ & $P$ value* & $P$ valuet & $P$ valuef \\
\hline Mean interval to echocardiography (y) & $1.8 \pm 1.1$ & $2.3 \pm 1.5$ & .07 & & \\
\hline Resting LV outflow gradient (mm $\mathrm{Hg}$ ) & $15(0,96)$ & $5(0,17)$ & $<.001$ & $<.001$ & .002 \\
\hline Provoked LV outflow gradient (mm Hg) & $42(0,125), n=50$ & $14(0,42), n=27$ & $<.001$ & $<.001$ & .004 \\
\hline Presence of systolic anterior motion of the mitral valve & $32 / 48(67 \%)$ & $12 / 41(29 \%)$ & $<.001$ & .006 & .01 \\
\hline Presence of moderate or more severe mitral valve regurgitation & $8 / 53(15 \%)$ & $6 / 45(13 \%)$ & .84 & .96 & .65 \\
\hline
\end{tabular}

$L V$, Left ventricular.

*Unadjusted.

$†$ Adjusted for significant baseline variables.

$\ddagger$ Adjusted for significant baseline variables and propensity score.

SAM was significantly associated with the baseline variables of greater left ventricular end-diastolic dimension and better NYHA functional class. After adjusting for these 2 baseline variables, SAM remained more likely in the alcohol ablation group (odds ratio, 4.02; $P=.006$ ), even after additional adjustment with propensity score $(P=.01)$. The presence of moderate or greater grade of mitral valve regurgitation was not significantly different between groups, even after adjusting for the significant baseline variables of presence of preprocedure arrhythmia and greater left ventricular end-systolic dimension $(P=.96)$ and additionally for propensity score $(P=.65)$. Similarly, the postprocedure prevalence and severity, usually trivial, of aortic insufficiency was not significantly different after ablation (37\%) versus myectomy (41\%).

An optimal composite outcome at follow-up was defined as survival, NYHA functional class I, no postprocedure pacemaker placement, and a follow-up resting left ventricular outflow gradient of less than $20 \mathrm{~mm} \mathrm{Hg}$. This was noted in $12(22 \%)$ patients in the alcohol ablation group and 35 $(73 \%)$ patients in the isolated myectomy group $(P<.001)$.

\section{Discussion}

There are no randomized prospective studies to compare the efficacy of alcohol ablation versus surgical myectomy. Only 3 publications compare the outcomes of both. ${ }^{11-13}$ Qin and colleagues, ${ }^{12}$ comparing 25 patients undergoing alcohol ablation with 26 patients undergoing myectomy, found myectomy to be more effective in reducing left ventricular outflow tract gradient immediately after the procedure and at 3 months' follow-up. Both procedures had similar effects on NYHA classification. The second study of Qin and colleagues ${ }^{13}$ compared the conduction abnormalities after intervention, demonstrating that ablation commonly results in right bundle branch block (62\%), whereas myectomy causes left bundle branch block (93\% of their patients). One implication of their observation is that patients with preprocedure conduction block will almost certainly need permanent cardiac pacing if they have right bundle branch block and are undergoing an operation or left bundle branch block and intending to have ablation. The one patient in our series who crossed over to myectomy after ablation did require pacing. Although pacing is less prevalent after myectomy in this series, the difference is not significant after adjusting for the prevalence of coronary artery disease. The absence of coronary disease in our patients undergoing isolated myectomy is due to the fact that those patients requiring coronary artery bypass were excluded from the isolated myectomy group. The prevalence of pacing is not different between the isolated myectomy and myectomy with concomitant operation groups ( $12.5 \%$ vs $12.7 \%)$, but the risk of pacing is higher after ablation (19\%; Figure 2).

Nagueh and associates ${ }^{14}$ compared 41 patients undergoing ablation treated at Baylor with 41 patients undergoing myectomy treated at the Mayo Clinic. In their series the hemodynamic and functional results at 1 year after the procedure were similar in both groups. In contrast, our patients undergoing myectomy had lower pressure gradients at rest and with provocation, better functional class, and less SAM (although similar mitral regurgitation) compared with the patients undergoing ablation. Naqueh and associates had additional information regarding exercise duration and volume of oxygen use peak and maximum, and these were also equivalent in both groups. Complications of intervention in Nagueh and associate's series include complete atrioventricular block, which was more common after ablation (22\% vs $2 \%$ ), and aortic insufficiency, which was more common after myectomy. Aortic insufficiency, mild or more severe, was not significantly different in our series.

Univariate analysis of our study patient demographics revealed that patients undergoing alcohol ablation were older, with more hypertension, better NYHA class, more coexistent coronary artery disease, and lesser degrees of septal hypertrophy compared with surgical patients, who had a higher incidence of angina, palpitations, and presyncopesyncope with a thicker interventricular septum. Statistical attempts to propensity match patients from both groups were unsuccessful, demonstrating that the 2 groups are 
inherently different. Propensity adjustment scores corrected these differences between the alcohol ablation and isolated myectomy groups. After propensity adjustment, patients treated with alcohol ablation had significantly higher postprocedural resting $(P=.002)$ and provokable outflow tract gradients $(P=.004)$, more SAM $(P=.01)$, and worse NYHA classification at most recent follow-up $(P=.05)$. The alcohol ablation group also had longer postprocedural length of stay $(P=.04)$, perhaps because of a learning curve related to this new procedure and delayed discharge while confirming the absence of atrioventricular block.

Mortality is statistically higher after alcohol ablation compared with that after isolated myectomy (or myectomy with concomitant procedures). All 3 cardiac-related deaths occurred in the ablation group. However, the number of patients in our study is small, with few deaths, and therefore this difference in survival needs to be confirmed. The combination of higher residual outflow tract gradients at rest and with provocation and the subendocardial scaring in patients receiving alcohol ablation might, however, increase the likelihood of postprocedural arrhythmia. ${ }^{14}$ Others have reported ventricular tachycardia complicating ablation. ${ }^{13}$

Significantly more patients achieved the defined optimal outcome after myectomy compared with that after alcohol ablation $(73 \%$ vs $22 \%, P=.001)$.

Results from this study might not be generally applicable; surgical myectomy is an established procedure at our institution, whereas the indications, technique, and care of patients undergoing alcohol ablation are new and evolving. We are comparing the performance of 2 procedures with differing patient referral patterns without randomization, and follow-up is relatively short, with small numbers of patients. The long-term effects of higher provokable gradients and scarring in the hypertrophic ventricle of patients undergoing ablation are unknown and will require ongoing careful patient follow-up. ${ }^{15}$

We have attempted to adjust the effect of differences in baseline patient characteristics using propensity scores. Our data suggest that the outcomes after isolated surgical myectomy are significantly better than those after alcohol ablation. There are sufficient data to justify a prospective multicenter trial of the 2 modalities of treatment to determine the optimal management of patients with the obstructive form of hypertrophic cardiomyopathy.

\section{References}

1. Maron MS, Olivotto I, Betocchi S, et al. Effect of left ventricular outflow tract obstruction on clinical outcome in hypertrophic cardiomyopathy. N Engl J Med. 2003;348:295-303.

2. Williams WG, Wigle ED, Rakowski H, et al. Results of surgery for hypertrophic obstructive cardiomyopathy. Circulation. 1987; 76(suppl):V104-8.

3. Maron BJ, McKenna WJ, Danielson GK, et al. American College of Cardiology/European society of Clinical Cardiology Clinical Expert
Consensus document on Hypertrophic Cardiomyopathy. J Am Coll Cardiol. 2003;42:1687-713.

4. Schulte HD, Bircks WH, Loesse B, Godehardt EA, Schwartzkopff B. Prognosis of patients with hypertrophic obstructive cardiomyopathy after transaortic myectomy. Late results up to 25 years. J Thorac Cardiovasc Surg. 1993;106:709-17.

5. Schonbeck MH, Brunner-La Rocca HP, Vogt PR, et al. Long-term follow-up in hypertrophic obstructive cardiomyopathy after septal myectomy. Ann Thorac Surg. 1998;65:1207-14.

6. Merrill WH, Friesinger GC, Graham TP, et al. Long-lasting improvement after septal myectony for hypertrophic obstructive cardiomyopathy. Ann Thorac Surg. 2000;69:1732-5.

7. Williams WG, Ralph-Edwards, AC, Wigle ED. Surgical management of hypertrophic cardiomyopathy. Cardiol Rev. 1997;5:40-9.

8. Woo A, Rakowski H, Ross J, et al. Percutaneous transluminal septal myocardial ablation for hypertrophic obstructive cardiomyopathy: initial results and clarification of mechanism of benefit [abstract]. Can J Cardiol. 1999;15:174D.

9. Kimmelstiel CD, Maron BJ. Role of septal ablation in hypertrophic obstructive cardiomyopathy. Circulation. 2004;109:452-5.

10. Bhagwandeen R, Woo A, Ross J, et al. Septal ethanol ablation for hypertrophic obstructive cardiomyopathy: early and intermediate results of a Canadian referral center. Can J Cardiol. 2003;19:912-7.

11. Wigle ED, Schwatz L, Woo A, Rakowski H. To ablate or operate? That is the question. J Am Coll Cardiol. 2001;38:1707-10.

12. Qin JX, Shiota T, Lever HM, et al. Outcome of patients with hypertrophic obstructive cardiomyopathy after percutaneous transluminal septal myocardial ablation and septal myectomy surgery. J Am Coll Cardiol. 2001;38:1994-2000.

13. Qin JX, Shiota T, Lever HM, et al. Conduction system abnormalities in patients with hypertrophic obstructive cardiomyopathy following septal reduction interventions. Am J Cardiol. 2004;93:171-5.

14. Nagueh SF, Ommen SR, Lakkis NM, et al. Comparison of ethanol septal reduction therapy with surgical myectomy for the treatment of hypertrophic obstructive cardiomyopathy. J Am Coll Cardiol. 2001; 38:1701-6.

15. Factor SM, Butany J, Sole MJ, Wigle ED, Williams WG. Pathologic fibrosis and matrix connective tissue in the subaortic myocardium of patients with hypertrophic cardiomyopathy. J Am Coll Cardiol. 1991; $17: 1343-51$.

\section{Discussion}

Dr Rene Pretre (Zurich, Switzerland). Dr Williams, you are to be congratulated for having convincingly shed light on the 2 therapeutic options that exist now for the treatment of hypertrophic cardiomyopathy, namely alcohol ablation and surgical myectomy. You have further performed a sound statistical analysis to compare comparable subgroups using, in the absence of randomization, adjustment for baseline patient characteristics and propensity score in multivariable regression analysis.

Overall, your results were good in both groups, although surgical intervention achieved better outcome, especially regarding hard facts, such as improvement of clinical status, need for subsequent pacemaker implantation, and reduction of the outflow tract obstruction and its inherent consequences. Follow-up is, however, relatively short. I have 3 questions for you.

The first question relates to the incidence of arrhythmia late after the procedure both at the atrial and the ventricular level. What was the incidence of atrial fibrillation late after the procedure, a complication that occurred steadily in our experience, up to $25 \%$ after 7 years, and also, have you performed Holter monitoring to detect dangerous ventricular arrhythmia late after surgical intervention that could justify the implantation of a defibrillator, for instance? 
Dr Williams. The question of arrhythmias in hypertrophic cardiomyopathy is an excellent one. Off the top of my head, I cannot recall what percentage had atrial fibrillation. There were 2 patients who underwent a maze procedure for chronic atrial fibrillation, but they are not in the comparative group because they had concomitant procedures. Otherwise, the prevalence of atrial fibrillation was not extensive.

What is of concern, of course, is ventricular tachycardia fibrillation and sudden death, and it is worrisome that there were 2 sudden unexpected deaths in the ablation group. We have concerns that the scar caused by the alcohol ablation might be arrhythmogenic over the longer period of time, particularly early after ablation, when these patients will often have a residual gradient, because it takes some time for that gradient to reach a low level.

Dr Prêtre. After having established a technique for alcohol ablation at your institution, have you observed a decrease in the proportion of patients sent to surgical intervention? This trend has been seen in some hospitals because of the pressure of the referring cardiologist, who wants a less-invasive, although less-efficient, operation.

Dr Williams. It was interesting and somewhat surprising to me that the percentage of patients referred for surgical intervention over this 5-year period did not change; it was around $61 \%$ referred for intervention. Half of the surgical referrals were because of concomitant diseases that needed to be treated. The other half were patients who could have gone either to ablation or surgical intervention. As a policy, both the interventionalist and the surgeons interviewed these patients, and then we allowed the patients to decide. Therefore we expected these 2 groups would be equivalent, but when we tried to propensity match them, it was clear there were important differences between the 2 groups, and therefore we were unable to use propensity matching and had to rely on the propensity score.

Dr Prêtre. Maybe a practical question. You reported residual left ventricular outflow tract obstruction after provocation of 42 $\mathrm{mm} \mathrm{Hg}$ after alcohol ablation and only $14 \mathrm{~mm} \mathrm{Hg}$ after surgical myectomy. If you have a young and physically active patient, what would be your recommendation? Would you do alcohol ablation and leave some kind of residual obstruction where the patient would reach this critical gradient and maintain hypertrophy of his left ventricle and remain at risk for sudden death, or would you suggest surgical myectomy on this individual?

Dr Williams. You are asking a surgeon whether he would recommend surgical intervention?

Dr Prêtre. You were soft in your conclusions stating that both therapies achieved acceptable results. Because you are in the process of decision making with your colleagues, I expect you would recommend surgical intervention in those active patients.

Dr Williams. Well, I think your question is an important one, and the reason for doing this analysis and presenting the information is that I think there is serious concern that the patients have better access to the catheterization laboratory than they do to the operating room. The conclusion that I make from this study is that it justifies doing a multicenter trial to demonstrate the benefits of one or the other treatment. There might be situations in which alcohol ablation is a better choice, but if you wanted to be certain that you were relieving the pressure gradient both at rest and at exercise and probably have a better long-term survival, then I think the data suggest very clearly that surgical myectomy is the better option.
Dr Richard J. Shemin (Boston, Mass). This was a nice study and very important. I am interested in 2 questions. In patients who are referred for alcohol ablation, do you know how often they find unfavorable septal anatomy, such as very small septals that cannot be cannulated or a single large septal perforator where a massive septal infarct might ensue? In these cases alcohol ablation would be aborted.

Dr Williams. To back up a little bit, I think that some of the patients were not referred for ablation if they had particularly thick septa. I think they were worried about infarcting too large an area, and these patients can get sick after ablation.

In terms of the ones who did go to the catheterization laboratory and were not ablated, of which there were 6,5 were because the anatomy was unfavorable and they could not either get into the right area or be certain they were infarcting the correct area. One of the 6 patients had an acute dissection of the coronary arteries and went to the operating room on an emergency basis, requiring a triple bypass and myectomy in spite of going into the catheterization laboratory with normal coronary arteries. Therefore this procedure did have some risk involved.

Dr Shemin. My second question relates to hospital resource use. Do you know whether they monitor these patients after the control infarct in the cardiac care unit for a few days, and what was the average length of stay of the patient receiving alcohol ablation?

Dr Williams. Interestingly, the length of stay in the patients undergoing alcohol ablation was longer than in the surgical patients, and when you adjust it by propensity score, it was longer by 4 days. Therefore from an in-hospital consumption standpoint, the surgical myectomy is more effective. Part of the long stay was their caution in undertaking a new procedure and making sure the physicians were not going to miss something when the patients went home.

Dr Alain Carpentier (Paris, France). I have 2 brief questions. You mentioned 5 cases of valve repair. Could you tell us what type of valve repair you were able to do?

Dr Williams. You are speaking of mitral valve repair?

Dr Carpentier. Yes.

Dr Williams. I think that the valve in hypertrophic obstructive cardiomyopathy almost always does not need to be repaired because it is secondary to the obstruction. When we relieve the obstruction, the mitral valve resumes its normal flow pattern and works quite well, and almost none of these patients had important regurgitation afterward. However, there is a small subset of patients with hypertrophic cardiomyopathy who concomitantly have mitral valve disease. These valves need to be repaired or, rarely, replaced by means of whatever technique is causing the regurgitation. It can be any of the various mitral lesions leading to regurgitation.

Dr Carpentier. There is another criterion to assess the quality of the results, which is whether you can diminish or even suppress the medical treatment. Could you elaborate on that and tell us whether you were able to discontinue medical treatment after myectomy and in which proportion?

Dr Williams. I do not have comparative data, and you have a very good point. I can tell you that most of the patients undergoing surgical myectomy are not receiving medication unless it is to suppress an arrhythmia. 
Dr Paul Kurlansky (Miami Beach, Fla). It is my understanding that the risk of sudden cardiac death in hypertrophic cardiomyopathy is not well correlated with symptomatic outflow obstruction, but what I was wondering is the following: Do you have any evidence from your work or that of others that surgical myectomy and relief of outflow tract obstruction has any effect on the long-term risk of sudden cardiac death in the patients with hypertrophic cardiomyopathy?

Dr Williams. Your question refers to the long-term survival with and without myectomy. I think Dr Maron had a very nice review of this in The New England Journal of Medicine about a year ago showing that long-term survival is related to the septal thickness, and we undertook a study of all of our patients undergoing myectomy to see whether outcomes were affected by myectomy.

Mortality after myectomy is very low and probably better than the natural history. The patients with thicker septa did not do better or worse in the long term. In other words, the benefit of surgical intervention seemed to be about the same, irrespective of whether the patient has a thin septum or a thick septum.

\section{Bound volumes available to subscribers}

Bound volumes of The Journal of Thoracic and Cardiovascular Surgery are available to subscribers (only) for the 2005 issues from the Publisher, at a cost of $\$ 134.00$ for domestic, $\$ 165.85$ for Canadian, and $\$ 155.00$ for international subscribers for Vol 129 (January-June) and Vol 130 (July-December). Shipping charges are included. Each bound volume contains a subject and author index.

The binding is durable buckram with the Journal name, volume number, and year stamped in gold on the spine. Payment must accompany all orders. Contact Elsevier Inc., Subscription Customer Service, 6277 Sea Harbor Dr, Orlando, FL 32887, USA; phone $800-654-2452$ or $407-345-4000$.

Subscriptions must be in force to qualify. Bound volumes are not available in place of a regular Journal subscription. 